\title{
(B) ULTRAVIOLET MISSIONS
}




\title{
THE EXTREME ULTRAVIOLET EXPLORER MISSION
}

\author{
STUART BOWYER \\ Center for Extreme Ultraviolet Astrophysics, \\ University of California, Berkeley, California 94720
}

\begin{abstract}
The Extreme Ultraviolet Explorer mission is described. The satellite is now scheduled to be launched in September, 1991. For the first six months, an all sky survey will be carried out covering 90 to $820 \AA$, or essentially the entire EUV bandpass. This EUV survey will be made in four bands, or colors, $\lambda \lambda 90-150 \AA ; 170-250 \AA, 400-600 \AA$ and $550-750 \AA$. A portion of the sky which is free from the normally intense $304 \AA$ geocoronal helium background will be surveyed at greater sensitivity; the wavelength coverage of this band is from 90 to $400 \AA$. Following the sky survey portion of the mission, spectroscopy of individual sources will be carried out. Three spectrometers employing novel variable line spaced gratings will provide spectra with $\sim 1 \AA$ resolution over the band from 90 to $800 \AA$. This spectroscopy will be carried out by guest observers chosen by NASA in a manner roughly analogous to the IUE guest observer program.
\end{abstract}

\section{Introduction}

The Extreme Ultraviolet Explorer (EUVE) mission, currently scheduled for launch in September 1991, is the culmination of some 25 years of effort at the University of California at Berkeley to develop the field of extreme ultraviolet (EUV) astronomy (defined here as astronomy from roughly 100 to $1000 \AA$ ). When I started this effort in the mid 1960's, there was universal agreement by the astronomical community that stellar observations at these wavelengths would not be possible because of the high opacity of the interstellar medium. Furthermore, astronomical instrumentation for the EUV was primitive to nonexistent. Solar EUV astronomy was carried out with photographic film as the detector of choice, and spark discharge tubes were the standard laboratory sources for EUV radiation. There were no calibrated standards for use at these wavelengths. We began by developing astronomical instrumentation for these wavelengths, which were then flown as part of my sounding rocket program (Henry et al. 1975a; Henry et al. 1975b; Henry et al. 1975c). We also developed laboratory calibration instrumentation. As an adjunct to this work we developed and deployed in space instrumentation to characterize the diffuse EUV background since this background was unknown and might limit astronomical measurements (Bowyer et al. 1974; Bowyer et al. 1977; Bowyer et al. 1981). It is interesting to note that this background has turned out to be of intrinsic interest (Chakrabarti et al. 1982; Paresce et al. 1983; Chakrabarti et al. 1983; Bowyer et al. 1983; Kumar et al. 1983).

This initial work led to and culminated in the flight of a Berkeley flux collector on the Apollo-Soyuz Mission in 1975. Although the instrumentation employed was crude by current standards and the observational program was quite limited, four astronomical sources of EUV radiation were discovered (Lampton et al. 1976; Margon et àl. 1976; Haisch et al. 1977; Margon et al. 1978). While the results obtained 
were of intrinsic scientific interest, their major impact was that they provided a vivid demonstration that EUV astronomy was, in fact, possible. Within a year or so thereafter, a NASA review panel identified the Berkeley EUVE mission as a prime mission for development and NASA chose this mission for implementation.

\section{EUVE Mission and Instrumentation}

The primary goal of the EUVE mission will be to carry out an all sky survey over the entire EUV band. This survey will be conducted in four subbands, or colors. It was originally expected that this would be the first survey in this band, but in the passage of time other EUV missions have been developed which will carry out at least partial surveys in this band. Most noteworthy in this regard is the British Wide Field Camera to be flown as part of the ROSAT mission. This mission (described elsewhere in this volume) will carry out a survey at the shortest EUV wavelengths. While it is certain that the majority of EUV sources will be observed at shorter wavelengths, it is clear that the sky should be mapped over the entire EUV band at least once. In addition, our experience with $\mathrm{x}$-ray astronomy, where at least five surveys of roughly equal sensitivity were conducted, has shown that multiple surveys bring out new and unexpected results.

The EUVE mission will also carry out a deeper survey over a limited portion of the sky. This data will provide insights into the types of sources which would be discovered in a more sensitive all sky survey. In specific would fainter sources be similar in character to the sources observed at higher intensities, or would an entirely new class of sources be evident?

What kinds of objects will EUVE detect? The answer is that no one knows. This is a true "explorer" mission; no surveys have been carried out and we must wait and see what nature has provided. It is generally agreed, however, that at least two classes of objects will certainly be detected: hot white dwarfs and coronally active stars. Estimates of the number of these objects which will be observed vary widely but generally fall in the range of 200 to 2000 objects. Hence it seems likely that the survey will at least detect a number of EUV sources which is equivalent to that detected by the first $\mathrm{x}$-ray surveys.

Finally, EUVE will carry out spectroscopy of the brighter sources discovered in the survey phase of the mission. This spectroscopy will be carried out exclusively by guest observers, similar in manner to the IUE guest observer program.

In Figure 1 I show the sensitivity of the instrumentation on the EUVE satellite. For comparison I show the spectrum of the hot white dwarf HZ43, the most intense EUV source now known. The all sky, all band surveys will be capable of detecting sources roughly one-hundredth as intense as HZ43. The deep survey is much more sensitive but covers only part of the EUV band and only a part of the celestial sphere. The sensitivity of the spectrometer depends on the integration time and the character of the source; I show in Figure 1 the sensitivity for a continuum source and one day's integration.

The telescopes for EUVE are shown in Figure 2. The total complement of telescopes consists of two Wolter-Schwarzschild Type I telescopes used to carry out the short wavelength bands of the survey and one Wolter-Schwarzschild Type II Tele- 


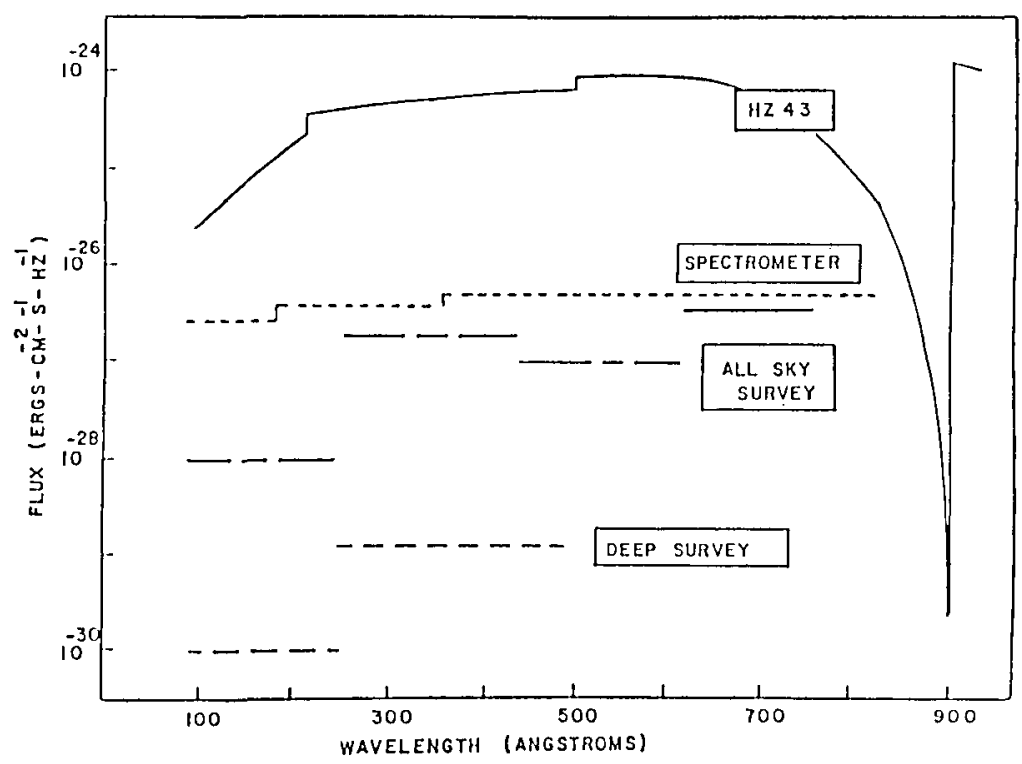

Fig. 1. The overall sensitivity of the EUVE instruments when combined with the anticipated sky integration times in the survey phase. The dotted line that is marked spectrometer. shows the sensitivity for a continuum source and one day's integration time.

scope for the longer wavelength band passes. The large graze angles in this design (Finley et al. 1988) prevents the (presumably more numerous) shorter wavelength sources from registering in the long wavelength band and compromising this part of the survey. A standard Wolter-Schwarzschild telescope is the collector for the 3 spectrometers and the deep survey.

The mirrors are made of metal and represent some fifteen years of development at Berkeley that began with mirrors initially developed for my sounding rocket program (Bowyer et al. 1985). The fabrication of these mirrors starts with aluminum forgings which undergo extensive pre- and post-machining thermal stabilization. The optical figure is cut utilizing the precision machining facilities of the Lawrence Livermore National Laboratories, and a final surface polish is employed to remove microscopic tool marks. The performance of these mirrors is exceptional. (Green et al. 1986). In Figure 3 I show the encircled energy of a point source as a function of encircling diameter for one of these mirrors. As can be seen, the half energy diameter is about 4 arcseconds, which is more than an order of magnitude better than any other metal mirror reported in the literature, and is almost a factor of two better than the Einstein glass mirror which cost well over an order of magnitude more to fabricate.

The band pass separation for the sky survey is provided by thin (300 to $3000 \AA$ thick) organic and metallic filters working in combination with the characteristics of the telescopes. We began development of these filters as part of my sounding rocket program (Chakrabarti et al. 1982; Jelinsky et al. 1983; Labov, Bowyer and Steele 1985; Hurwitz, Labov and Chakrabarti 1985). On the basis of 


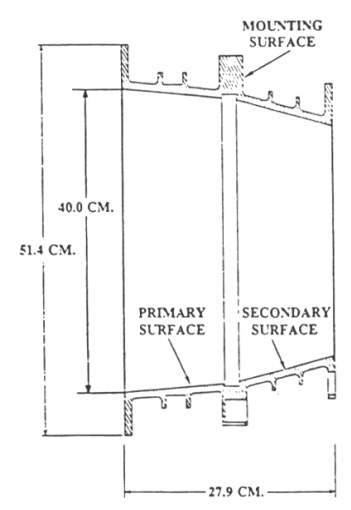

TYPE I SCANNER

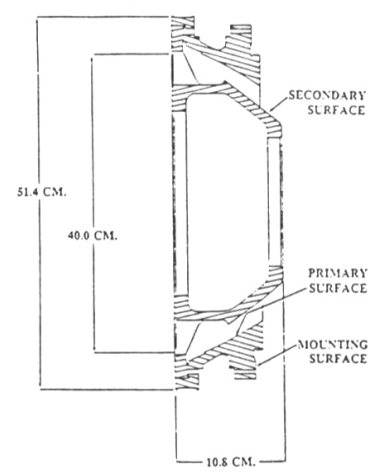

TYPE II SCANNER



DEEP SURVEY/SPECTROMETER

Fig. 2. Cross sectional schematic diagrams of the three types of mirrors employed.

what was known in 1975 we proposed filters of (a) parylene (a carbon/hydrogen polymer), (b) aluminum overcoated with carbon, (c) antimony, and (d) tin. Upon initiation of the EUVE Program we intensified our efforts in this area both in the range of filter materials explored and in testing (Vedder et al. 1989). A summary of some of our efforts in this regard is shown in Table I.

The response of the filter complement employed in EUVE is shown in Figure 4. These filters are rugged and stable, separate the EUV band into reasonable subbands, and minimize (for the most part) the background due to non-stellar sources.

The detectors employed are photon counting multichannel plate intensifiers with wedge-and-strip encoding. This type of detector was invented in our laboratory by Dr. Michael Lampton and co-workers (Martin et al. 1981) and was extensively improved and space qualified by Dr. Oswald Siegmund and co-workers (Siegmund et al. 1986). The detectors in the EUVE provide $1680 \times 1680$ RMS independent resolution elements, are linear to better than $1 / 2 \%$, and possess a variety of additional attributes (they are stable, rugged, and solar blind; they have graceful degradation with high counting rate, plus more). 




Fig. 3. Encircled energy for the deep survey/spectrometer telescope. This is well over an order of magnitude better than other metal mirrors and is a factor of two better than the Einstein mirrors.

TABLE I

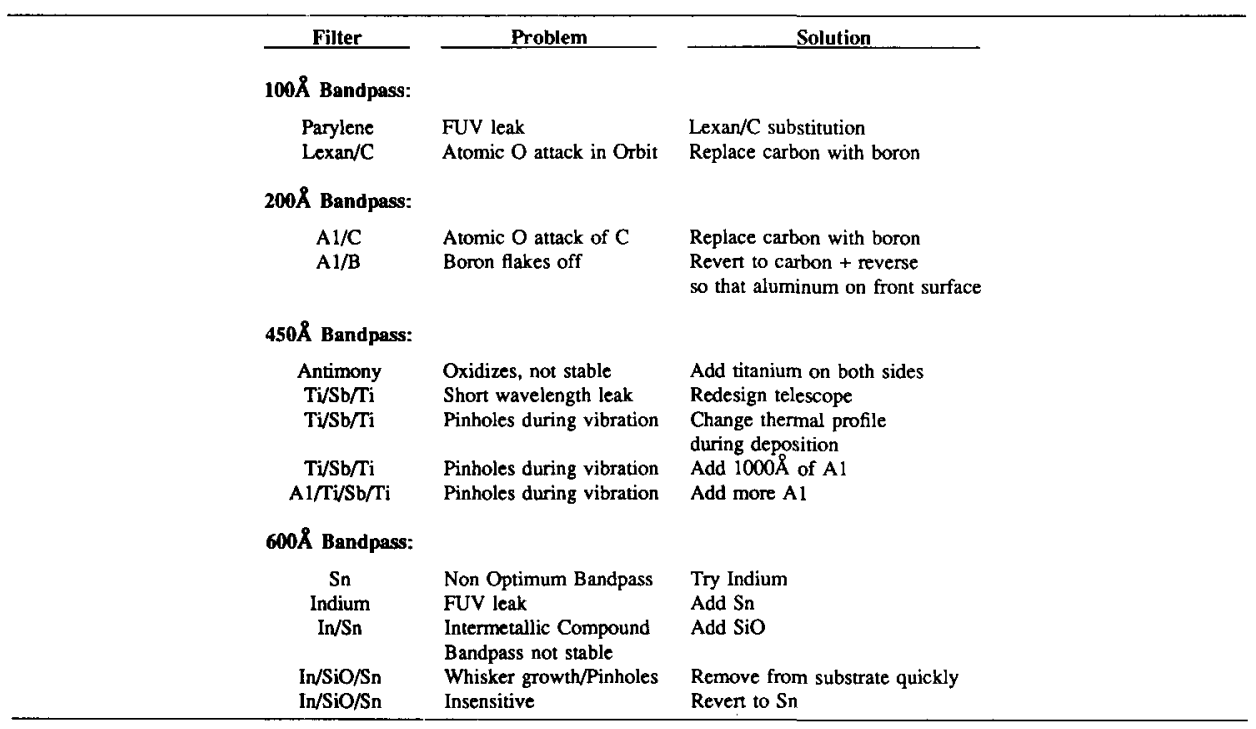




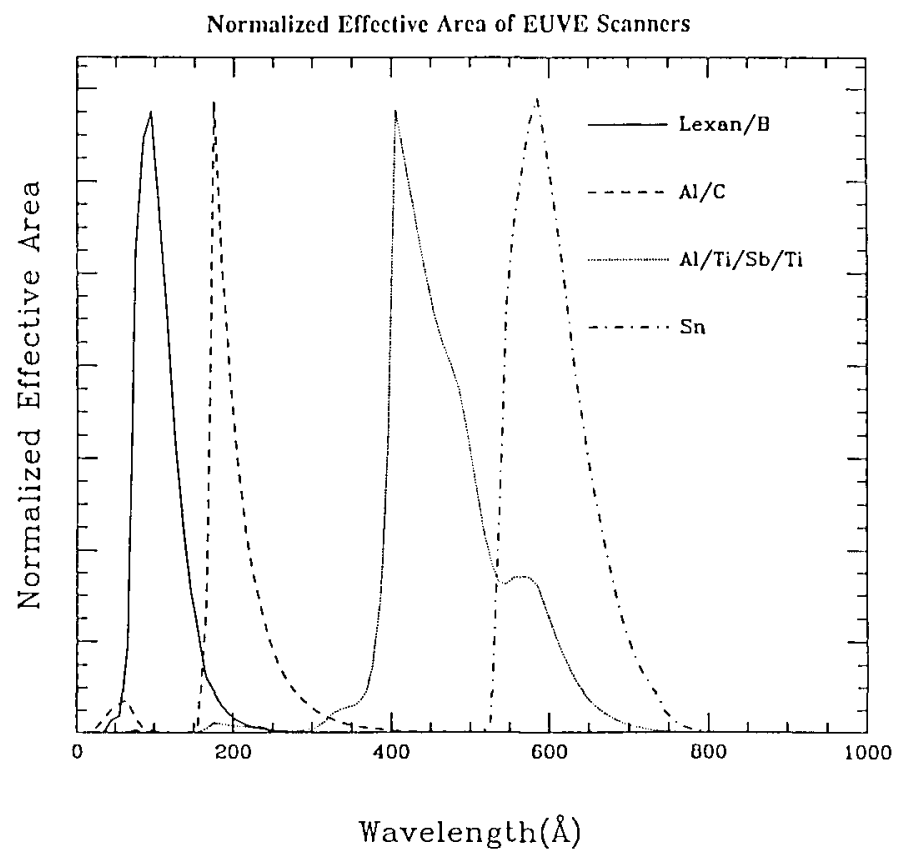

Fig. 4. The filter complement employed in defining the subbands of the all sky survey.

We have carried out an extensive effort to develop stable, high quantum efficiency photocathodes for the EUV, and to optimize their incorporation in the detectors (Martin and Bowyer 1982; Everman et al. 1987; Siegmund et al. 1986; Siegmund et al. 1988). In Figures 5a and 5b I show the quantum efficiency of two of the materials which have been developed and characterized in this effort.

The telescopes have been extensively parameterized in our calibration facility (Jelinsky and Malina 1988). In Figure 6 I show one of the images obtained in the calibration sequence. The linear enhanced areas are an image of a laboratory source as the articulated calibration mounting replicates the movement of an astronomical source as it will be observed in the normal observing mode. The images are offset as the satellite spin vector moves to follow the change in the sun-earth vector. The lower portion of the image plane appears blank because the filter in this half of the detector is (almost) opaque to the radiation employed.

In Figure 7 I show the source after it has been reconstructed from the data in the upper half of the image shown in Figure 5. Not surprisingly, the source is quite obvious. In Figure 8 I show the image reconstructed from the data in the lower half of the image plane. Although the source was certainly not obvious in the raw data shown in Figure 6, it stands out clearly in this reconstruction.

The spectrometer is an entirely new design invented at Berkeley (Hettrick and Bowyer 1983). This spectrometer uses variable line spaced gratings and provides a substantial number of advantages for spectroscopy with grazing incidence optics. First, it is highly efficient in that it requires a minimum number of grazing incidence reflections. Second, the image plane is nearly normal to the direction of the principal 

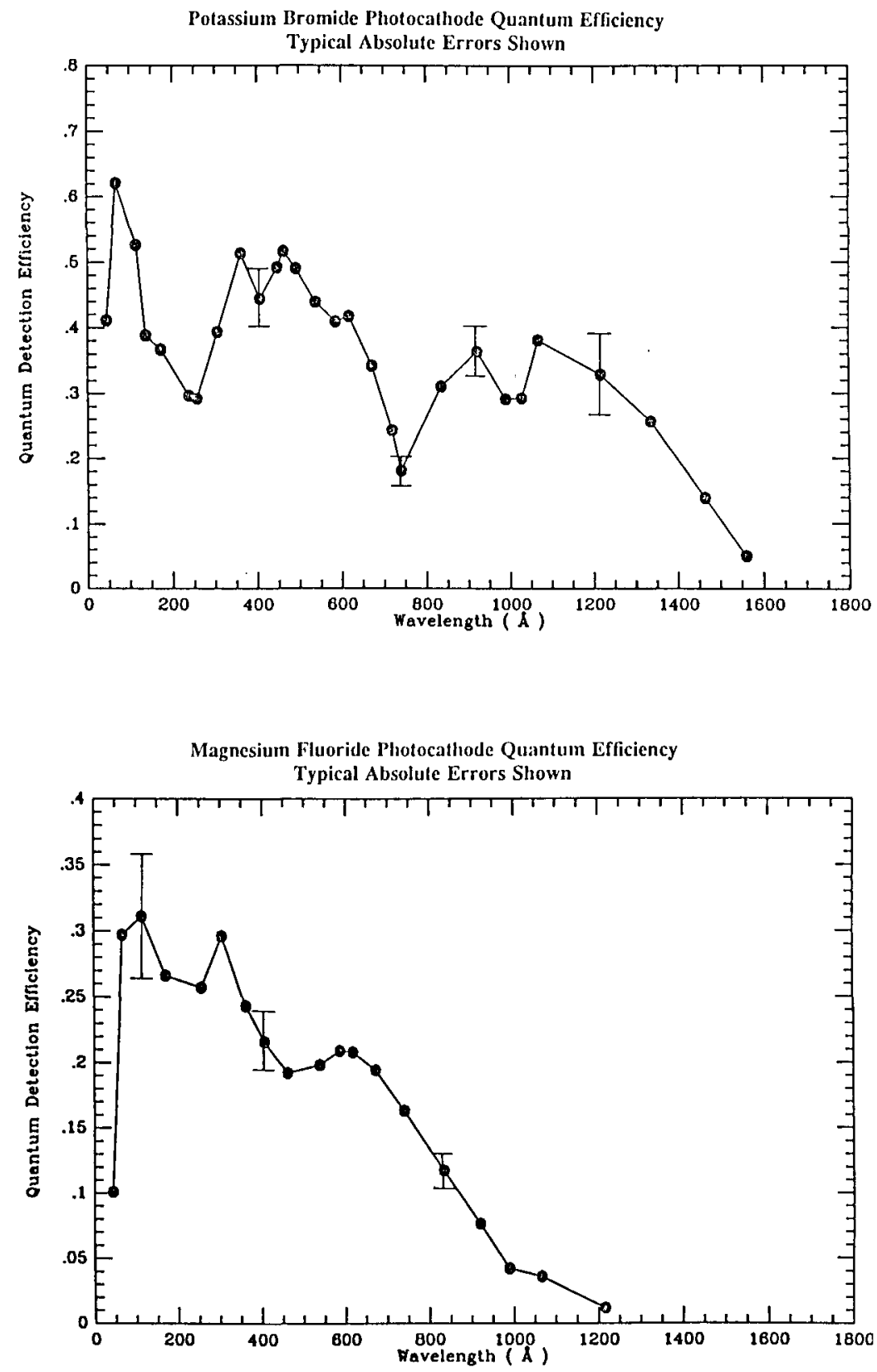

Fig. 5. Quantum efficiency of potassium bromide and magnesium fluoride as a function of wavelength. 
TABLE II

Grating Characteristics

\begin{tabular}{|l|l|l|l|}
\hline & \multicolumn{1}{c|}{ Short } & \multicolumn{1}{c|}{ Medium } & \multicolumn{1}{c|}{ Long } \\
\hline Line Density $\left(\mathrm{mm}^{-1}\right)$ & $1675-3550$ & $830-1750$ & $415-875$ \\
Average Plate Scale $(\AA / \mathrm{mm})$ & 2.4 & 4.8 & 9.6 \\
Blaze Angle & $3^{\circ}$ & $3^{\circ}$ & $3^{\circ}$ \\
Coating & Rhodium & Platinum & Platinum \\
Filters & Lex/B & Al and Lex/B & Al and Al/C \\
\hline
\end{tabular}

ray. Finally, it is readily adaptable to grazing incidence optics and provides the opportunity for optimizing compactness, plate scale, resolution, etc. The concept of this spectrometer is illustrated in Figure 9.

This concept has been actualized in the EUVE deep survey/spectrometer assembly (Martin et al. 1985). This assembly is shown in Figure 10. Three spectrometers are employed so the gratings will be reasonably on-blaze over the wavelength range from 80 to $800 \AA$. The characteristics of the gratings used in these spectrometers are listed in Table II.

The spectrometers on EUVE have been extensively calibrated. The results of one of the calibrations are shown in Figure 11. This data was obtained at a synchrotron facility and characterizes the continuum response of the grating (Jelinsky et al. 1988). The structure exhibited in the response of the grating is due to the thin metallic overcoat on the grating surface.

We have generated simulated spectra of the response of the telescope/spectrometer to astronomical objects using models of the objects folded through the calibrated response of our instrument. In Figure $12 \mathrm{I}$ show the raw spectrum of a continuum source, the hot white dwarf G191-B2B, and in Figure 13 I show the spectrum of Capella assuming its chromospheric emission is enhanced in intensity by the ratio x-ray $\left.\right|_{\text {Capella }} / \mathrm{x}-\left.\mathrm{ray}\right|_{\text {Sun }}$. Both simulations include the effects of interstellar absorption and detector background and assume a 40,000 second observation.

The spectrum of G191-B2B shows the high photometric accuracy expected to be obtained with brighter continuum sources in reasonable observation times. The spectrum of Capella suggest the variety of lines which we might hope to observe in coronally active stars. This is especially noteworthy since extensive work on the sun has demonstrated the power of EUV emission line studies as diagnostics of chromospheric plasmas.

Finally, in Figure 14, I show the simulated spectrum of a continuum source with $1 / 20$ the intensity of HZ43 and an intervening column of $10^{18}$ hydrogen atoms and a neutral hydrogen to helium ratio as indicated.

The absorption edge at $504 \AA$ due to neutral helium in the interstellar medium is clearly visible even in the weak source modeled here. These simulations demonstrate the power of the EUVE spectrometer in determining this parameter. Green, Jelinsky and Bowyer (1990) have carried out a measurement of this type with a 
rocket borne payload to provide constraints on the ionization state of the nearby interstellar medium in one view direction. With EUVE we can expect to determine this parameter in a significant number of view directions.

\section{Acknowledgements}

The development of the Extreme Ultraviolet Explorer has been a cooperative effort involving a substantial number of people at NASA and UCB. I especially acknowledge the contributions of Roger F. Malina, Michael Lampton, Oswald Siegmund, Pat Jelinsky, Herman Marshall, John Vallerga, David Finley, and Peter Vedder in the development of these instruments and in the preparation of this manuscript. This work has been supported by NASA contract NAS5-29298.

\section{References}

Bowyer, S., Freeman, J., Paresce, F., and Lampton, M.: 1977, Appl. Opt. 16, 756

Bowyer, S., Green, J., Finley, D., and Malina, R.: 1985, in Diamond Turned Grazing Incidence Mirrors for the Extreme Ultraviolet: Ten Years of Fabrication and Performance Results, ed(s)., , Proceedings of the NASA Grazing Incidence Optics Workshop, Annapolis, Maryland

Bowyer, S., Kimble, R., Paresce, F., Lampton, M., and Penegor, G.: 1981, Appl. Opt. 20, 477

Bowyer, S., Kumar, S., Paresce, F., and Lampton, M.: 1974, Appl. Opt. 13, 575

Bowyer, S., Paresce, F., Chakrabarti, S., and Kimble, R. A.: 1983, J. Geophys. Res. 88, 10247

Chakrabarti, S., Bowyer, S., Paresce, F., Franke, J., and Christensen, A.: 1982, Appl. Opt. 21, 3417

Chakrabarti, S., Paresce, F., Bowyer, S., Chiu, Y., and Aikin, A.: 1982, Geophys. Res. Letters 9,151

Chakrabarti, S., Paresce, F., Bowyer, S., Kimble, R., and Kumar, S.: 1983, J. Geophys. Res. 88, 4898

Finley, D. S., Jelinsky, P., Bowyer, S., and Malina, R.F.: 1988, Appl. Opt. 27, 1476

Green, J., Finley, D., Bowyer, S., and Malina, R. F.: 1986, The Extreme Ultraviolet Explorer: Optics Fabrication and Performance 628, Proc. SPIE

Green, J., Jelinsky, P., and Bowyer, S.: 1990, Ap.J. 359, to appear

Haisch, B. M., Linsky, J. L., Lampton, M., Paresce, F., Margon, B., and Stern, R.: 1977, Ap. J. 213, L119

Henry, P., Cruddace, R., Paresce, F., Bowyer, S., and Lampton, M.: 1975A, Ap. J. 195, 107

Henry, P., Cruddace, R., Paresce, F., Lampton, M., and Bowyer, S.: 1975B, Rev. Sci. Instrum., 46, 355

Henry, P., Cruddace, R., Lampton, M., Paresce, F., and Bowyer, S.: 1975C, Ap. J. Letters, 197, L117

Hettrick, M. C. and Bowyer, S.: 1983, Appl. Opt. 22, 3921

Hurwitz, M., Labov, S., and Chakrabarti, S.: 1985, Appl. Opt. 24, 1735

Jelinsky, P., Jelinsky, S., Miller, A., Vallerga, J., and Malina, R.F.: 1988, in L. Golub, ed(s)., Synchrotron Radiation Calibration of the EUVE Variable Line Spaced Diffraction Gratings at the NBS SURF II Facility, 356,

X-Ray Instrumentation in Astronomy II, Proc. SPIE 982 
Jelinsky, P., Martin, C., Kimble, R., Bowyer, S., and Steele, G.: 1983, Appl. Opt. 22, 1227 Kumar, S., Chakrabarti, S., Paresce, F., and Bowyer, S.: 1983, J. Geophys. Res. 88, 9271 Labov, S., Bowyer, S., and Steele, G.: 1985, Appl. Opt. 24, 576

Lampton, M., Margon, B., Paresce, F., Stern, R., and Bowyer, S.: 1976, Ap. J. Letters 203, L71

Margon, B., Lampton, M., Bowyer, S., Stern, R., and Paresce, F.: 1976, Ap. J. Letters 210, L79

Margon, B., Szkody, P., Bowyer, S., Lampton, M., and Paresce, F.: 1978, Ap. J. 224, 167

Martin, C. and Bowyer, S.: 1982, Appl. Opt. 21, 4206

Martin, C., Jelinsky, P., Lampton, M., Malina, R. F., and Anger, H. O.: 1981, Rev. Sci. Instrum. 52, 1967

Martin, C., Mrowka, S., Bowyer, S., Malina, R.F.: 1985, in The Extreme Ultraviolet Explorer Spectrometer: Performance Characteristics Based on Development of the Collimator, Variable Line Space Gratings, Telescope and Detectors, ed(s)., 284, Proc. SPIE 597 ,

Paresce, F., Chakrabarti, S., Bowyer, S., Kimble, R., and Kumar, R.: 1983, J. Geophys. Res. 88, 4905

Siegmund, O.H.W., Everman, E., Vallerga, J.V., and Lampton, M.: 1988, Appl. Opt. 27, 1568

Siegmund, O.H.W., Everman, E., Vallerga, J.V., Sokolowski, J., and Lampton, M.: 1987, Appl. Opt. 26, 3607

Siegmund, O.H.W., Lampton, M., Bixler, J., Chakrabarti, S., Vallerga, J., Bowyer, S., and Malina, R.F.: 1986, J. Opt. Soc. Am.-A 3, 2139

Siegmund, O.H.W., Vallerga, J.V., Everman, E., Labov, S., Bixler, J.: 1986, in High Quantum Efficiency Opaque CsI Photocathodes for the Extreme and Far Ultraviolet, ed(s)., 117, Proc. SPIE 687,

Vedder, P. W., Vallerga, J. V., Siegmund, O.H.W., Gibson, J., and Hull, J.: 1989, in The Filters for the Extreme Ultraviolet Explorer: Calibration and Lifetesting Results, ed(s)., 392, Proc. SPIE 1159,

B. Welsh, Jelinsky, P. and Malina, R. F.: 1988, in L. Golub, ed(s)., The Berkeley Extreme Ultraviolet Calibration Facility, X-Ray Instrumentation in Astronomy II, Proc. SPIE 982,335 


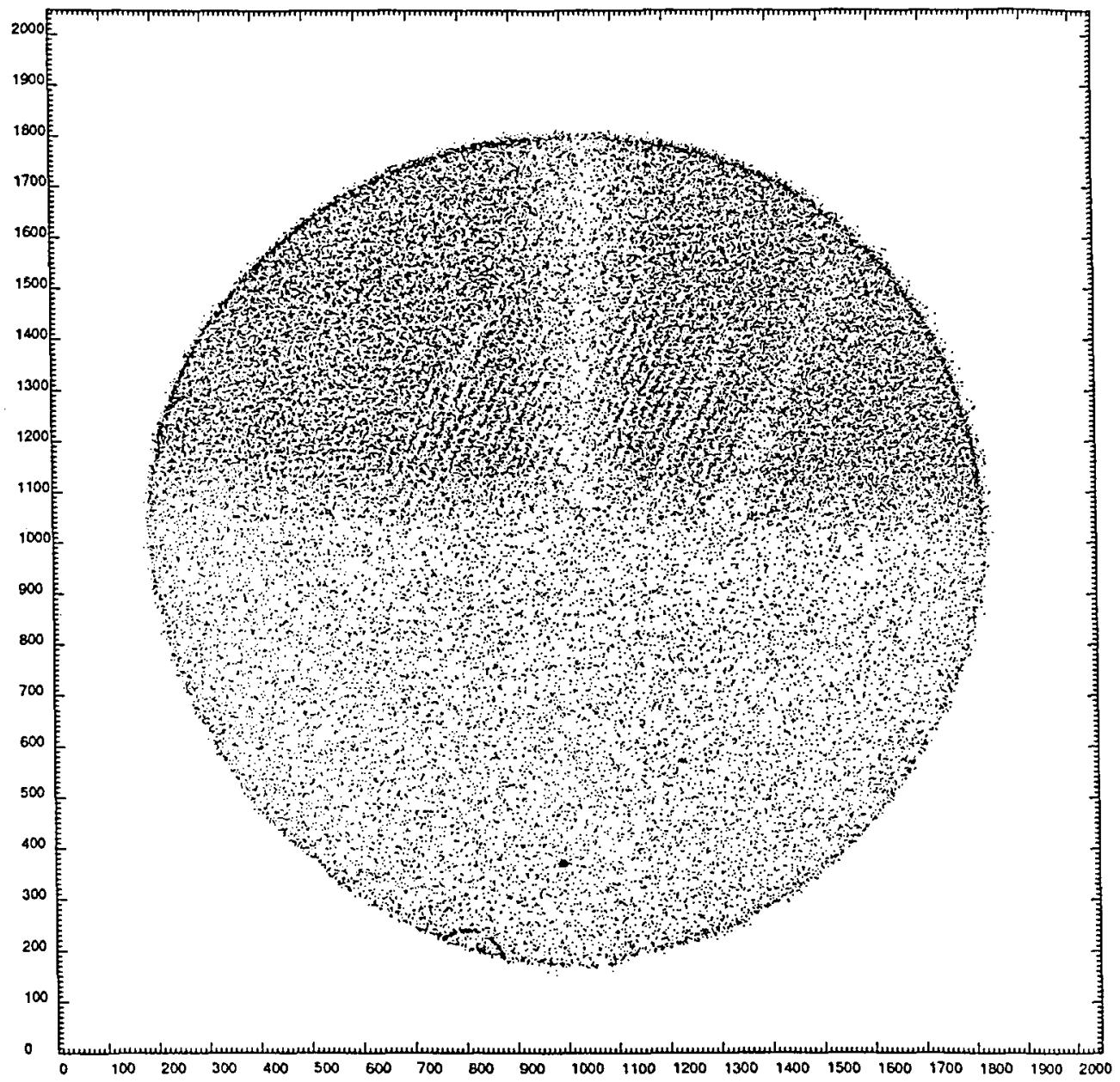

Fig. 6. Laboratory simulation of a source as it will appear in the normal operation mode in space. The thin linear structures are the source traversing the image plane as the telescope scans the sky. These are offset each orbit because of the progressive change in the sun-earth vector with time. The lower half of the image plane appears blank because of the effect of the filter which blocks radiation at this wavelength. 




Fig. 7. A reconstruction of the data obtained from the top half of the image shown in Figure 6. 


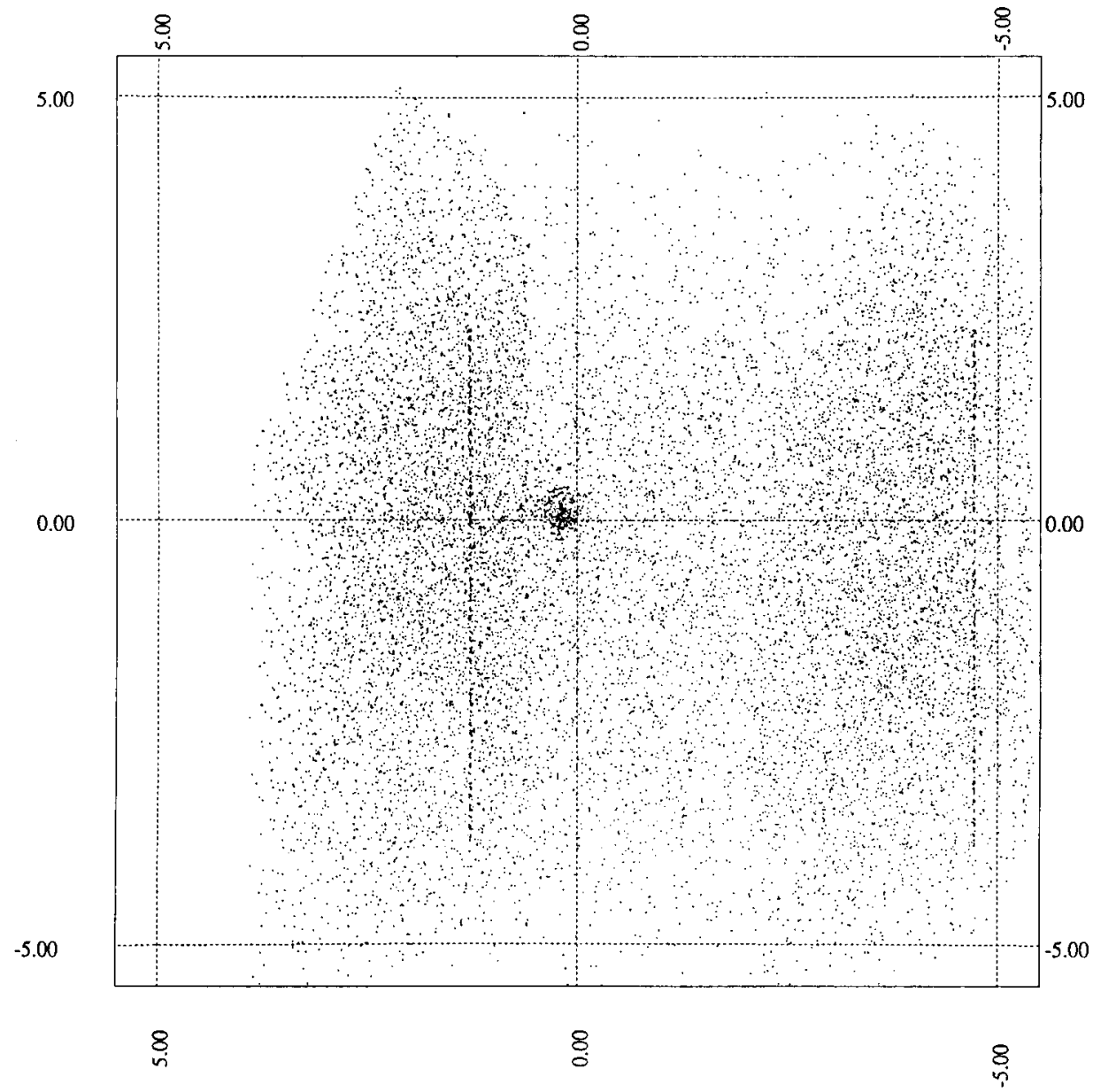

Fig. 8. A reconstruction of the data obtained from the bottom half of the image shown in Figure 6. 




Fig. 9. A schematic illustration of the concept of the variable line space grazing incidence spectrometer.

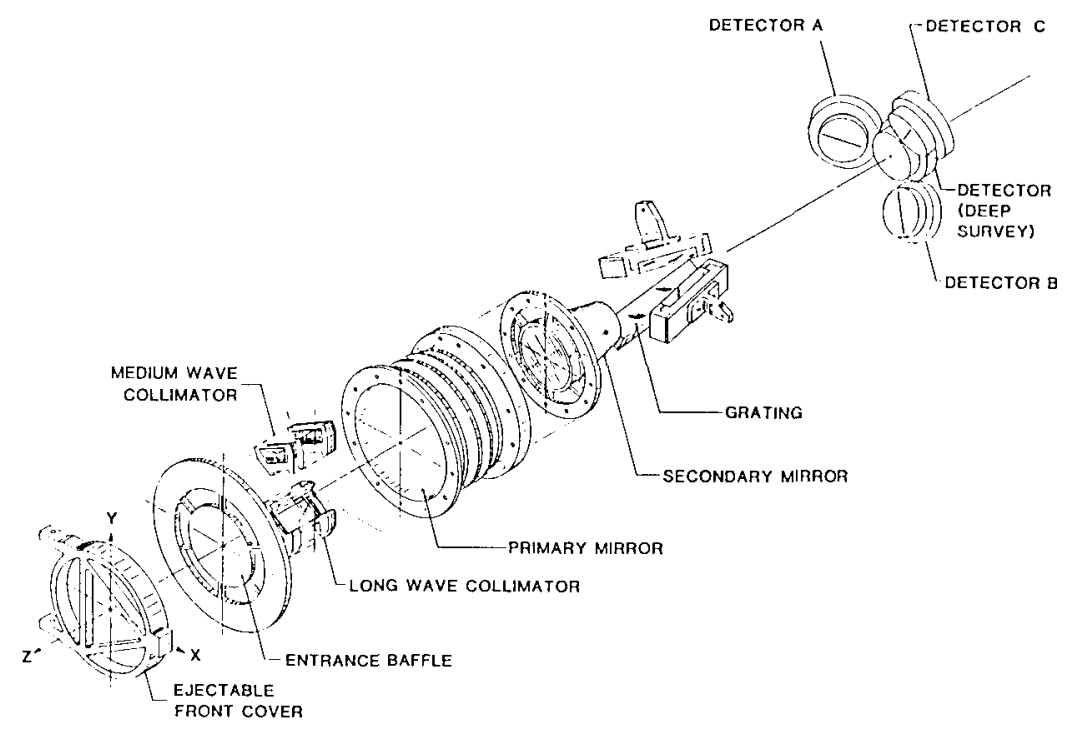

Fig. 10. An exploded schematic of the spectrometer on EUVE. 


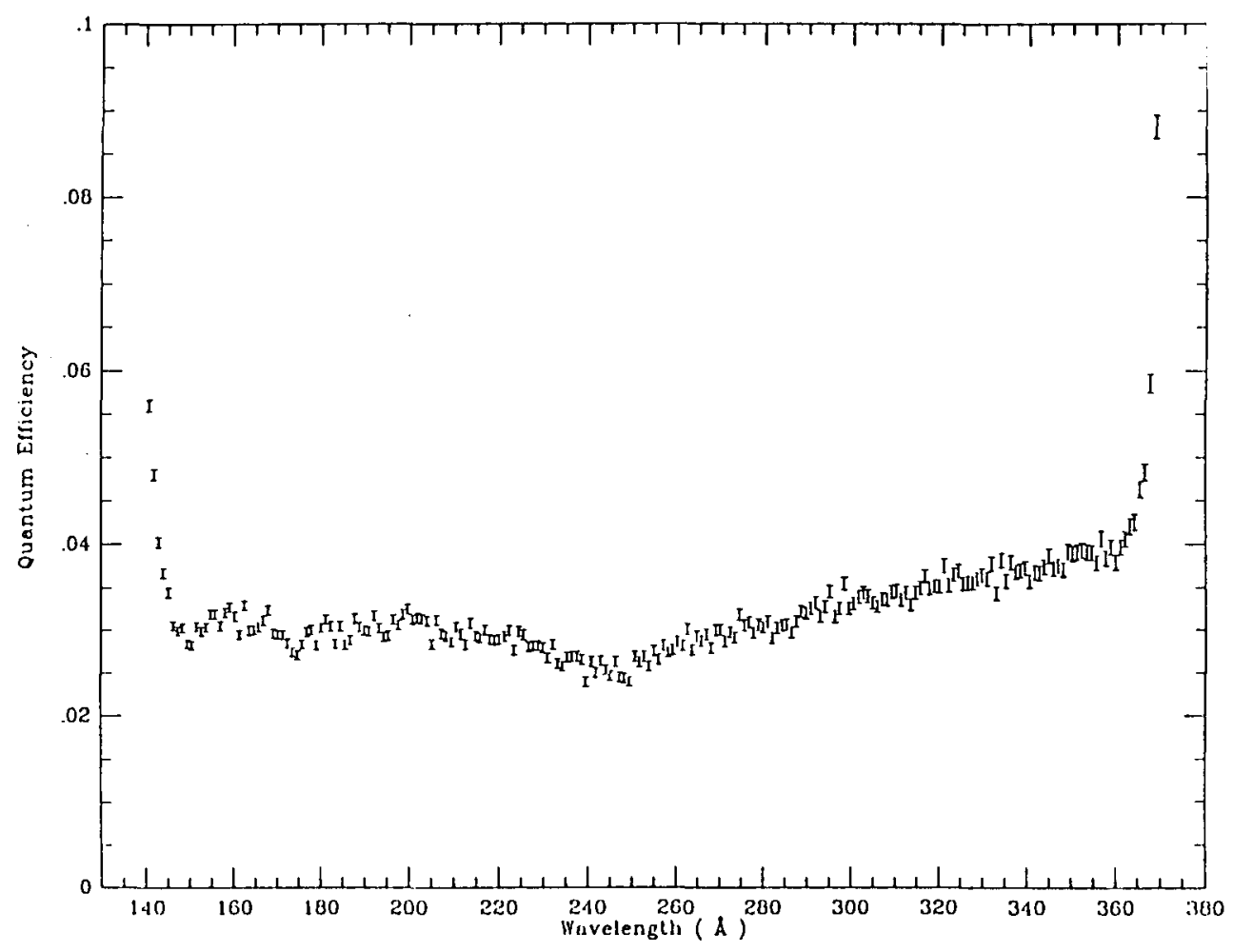

Fig. 11. The response of one of the gratings to continuum radiation. 
Simulated Spectrum of G191-B2B

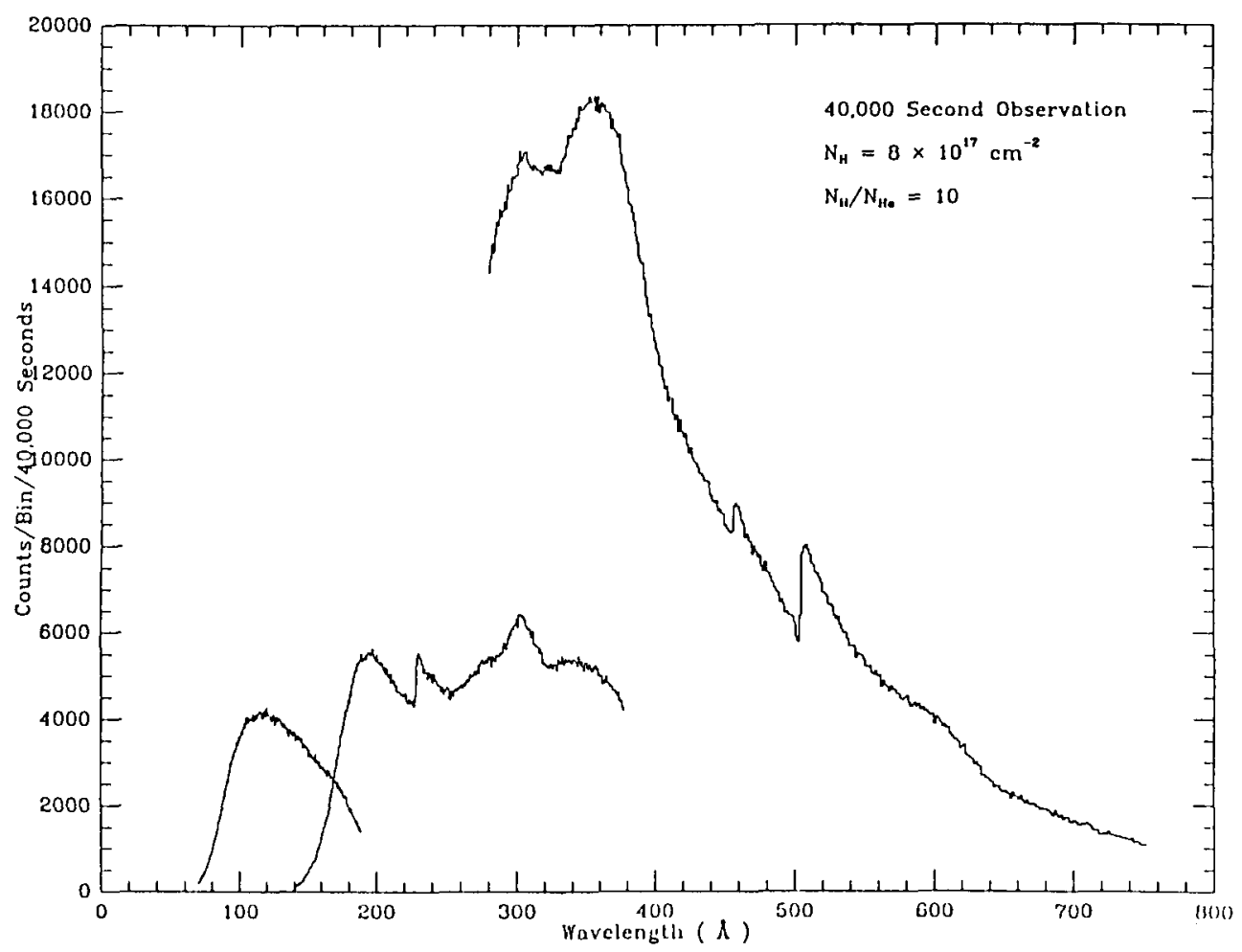

Fig. 12. Simulated spectrum of the hot white dwarf G19B2B as observed for 40,000 seconds. The effects of absorption of the ISM are included.

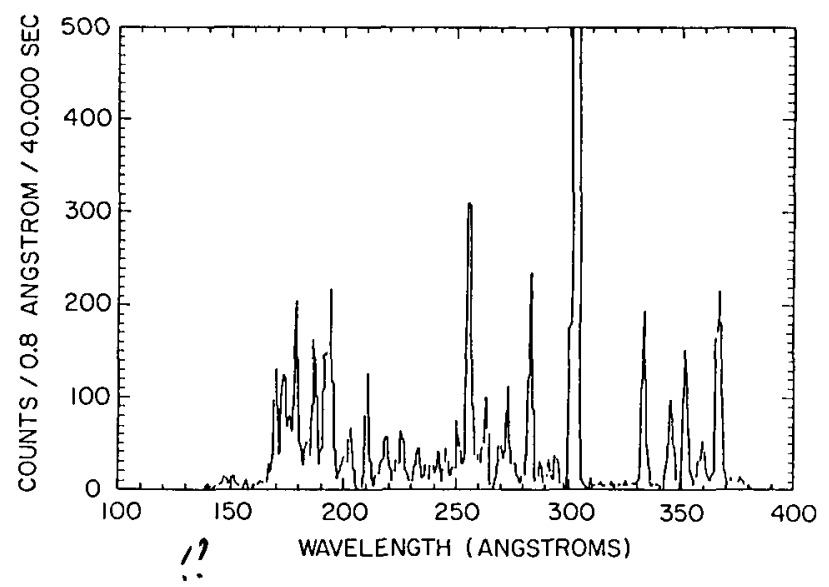

Fig. 13. Model of Capella's spectrum as observed for 40,000 seconds. 

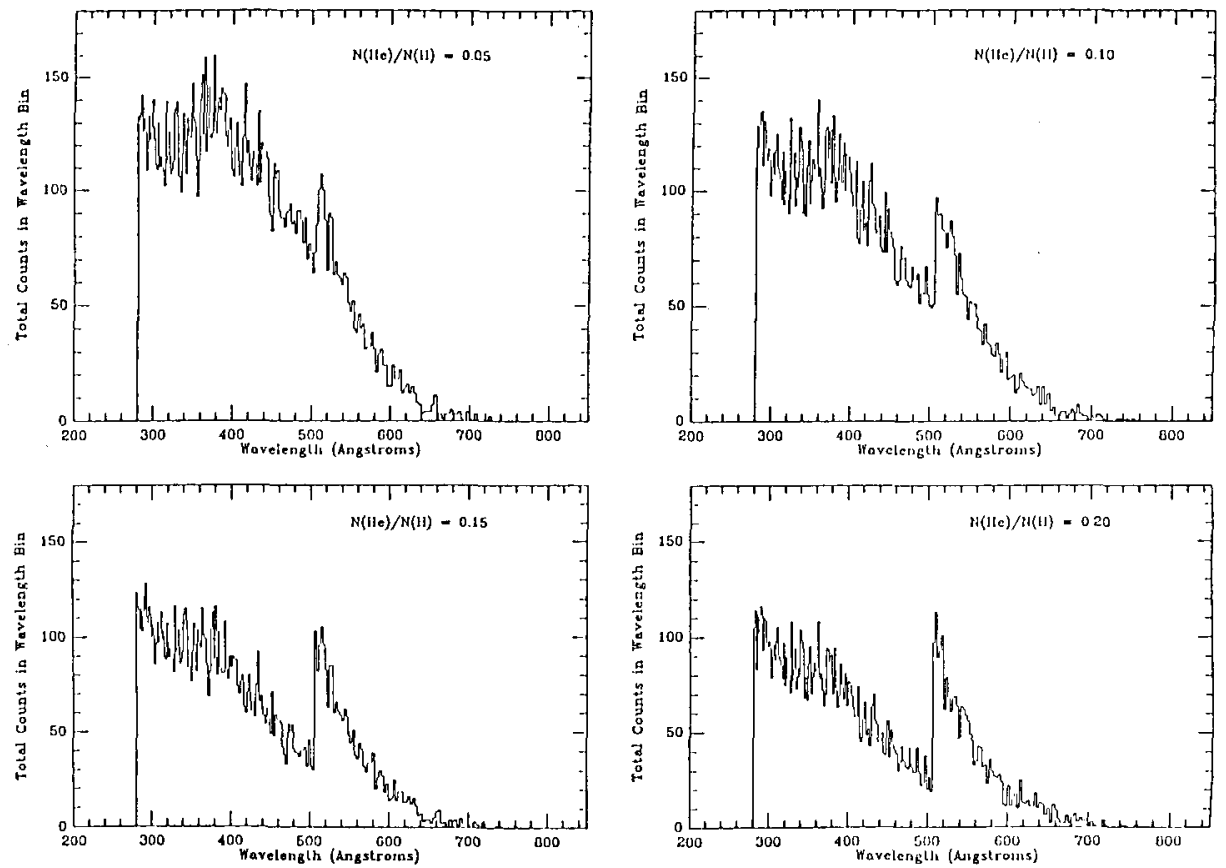

Fig. 14. The simulated response of a source with $1 / 20$ the intensity of HZ43 with an intervening column of $10^{18}$ hydrogen atoms and a neutral hydrogen to helium ratio as indicated. 